OPEN ACCESS

Edited by:

Jianfeng Pei,

Peking University, China

Reviewed by:

Youjun Xu,

Peking University, China

Chittaranjan Tripathy,

Walmart Labs, United States

*Correspondence:

Daniil Polykovskiy

daniil@insilico.com

${ }^{\dagger}$ These authors have contributed equally to this work

Specialty section:

This article was submitted to Translational Pharmacology,

a section of the journal

Frontiers in Pharmacology

Received: 11 November 2019

Accepted: 25 February 2020

Published: 17 April 2020

Citation:

Shayakhmetov R, Kuznetsov $M$, Zhebrak A, Kadurin A, Nikolenko S, Aliper A and Polykovskiy D (2020)

Molecular Generation for Desired

Transcriptome Changes With

Adversarial Autoencoders.

Front. Pharmacol. 11:269.

doi: 10.3389/fphar.2020.00269

\section{Molecular Generation for Desired Transcriptome Changes With Adversarial Autoencoders}

\author{
Rim Shayakhmetov ${ }^{1+}$, Maksim Kuznetsov ${ }^{1+}$, Alexander Zhebrak ${ }^{1}$, Artur Kadurin ${ }^{1}$, \\ Sergey Nikolenko ${ }^{1,2}$, Alexander Aliper ${ }^{1}$ and Daniil Polykovskiy ${ }^{1 *}$ \\ ${ }^{1}$ Insilico Medicine, Hong Kong, Hong Kong, ${ }^{2}$ Neuromation OU, Tallinn, Estonia
}

Gene expression profiles are useful for assessing the efficacy and side effects of drugs. In this paper, we propose a new generative model that infers drug molecules that could induce a desired change in gene expression. Our model-the Bidirectional Adversarial Autoencoder-explicitly separates cellular processes captured in gene expression changes into two feature sets: those related and unrelated to the drug incubation. The model uses related features to produce a drug hypothesis. We have validated our model on the LINCS L1000 dataset by generating molecular structures in the SMILES format for the desired transcriptional response. In the experiments, we have shown that the proposed model can generate novel molecular structures that could induce a given gene expression change or predict a gene expression difference after incubation of a given molecular structure. The code of the model is available at https://github.com/ insilicomedicine/BiAAE.

Keywords: deep learning, generative models, adversarial autoencoders, conditional generation, representation learning, drug discovery, gene expression

\section{INTRODUCTION}

Following the recent advances in machine learning, deep generative models found many applications in biomedicine, including drug discovery, biomarker development, and drug repurposing (Mamoshina et al., 2016; Zhavoronkov, 2018). A promising approach to drug discovery is conditional generation, where a machine learning model learns a distribution $p(x \mid y)$ of molecular structures $x$ with given property $y$. Such models can generate molecules with a given synthetic accessibility, binding energy, or even activity against a given protein target (Kadurin et al., 2016; Polykovskiy et al., 2018a).

In this paper, we studied how conditional models scale to a more complex biological property; specifically, we have studied how drug incubation influences gene expression profiles. Using the LINCS L1000 (Duan et al., 2014) dataset, we build a joint model $p(x, y)$ on molecular structures $x$ and induced gene expression changes $y$.

In many conditional generation tasks, $x$ completely defines $y$. For example, molecular structure completely defines its synthetic accessibility score. For our task, however, some transcriptome changes are unrelated to the drug effect on cells, and we cannot infer them only from an incubated drug. 
We propose a new model-the Bidirectional Adversarial Autoencoder-that learns a joint distribution $p(x, y)$ of objects and conditions. The model decomposes objects and their properties into three feature parts: shared features $s$ common to both $x$ and $y$; exclusive features $z_{x}$ relevant only to $x$ and not $y$; and exclusive features $z_{y}$ relevant only to $y$ and not $x: p(x, y)=p\left(s, z_{x}, z_{y}\right)$. For the transcriptomes and drugs, shared features $s$ may contain pharmacophore properties, target protein information, binding energy, and inhibition level; exclusive features $z_{x}$ may describe the remaining structural information; and $z_{y}$ may represent unrelated cellular processes. As features $s$ are common to both $x$ and $y$, the model can extract them from both $x$ and $y$.

The paper is organized into sections: Related Work surveys related work; Models presents the proposed Bidirectional Adversarial Autoencoder; Experimental Evaluation compares and validates the models on two datasets: the toy Noisy MNIST dataset of hand-written digits and LINCS L1000 dataset of small molecules with corresponding gene expression changes; and Conclusion concludes the paper.

\section{RELATED WORK}

Conditional generative models generate objects $x$ from a conditional distribution $p(x \mid y)$, with $y$ usually being limited to class labels. The Adversarial Autoencoder (AAE) (Makhzani et al., 2015) consists of an autoencoder with a discriminator on the latent representation $z$ that tries to make the latent space distribution indistinguishable from a prior distribution $p(z)$; its conditional extension-Supervised AAE (Makhzani et al., 2015)—works well for simple conditions but can violate the conditions in other cases (Polykovskiy et al., 2018b). Conditional Generative Adversarial Networks (CGAN) (Mirza and Osindero, 2014) supplied the condition as an auxiliary input to both generator and discriminator. Perarnau et al. (2016) inverted CGANs, allowing us to edit images by changing the labels $y$. In FusedGAN (Bodla et al., 2018), a GAN generated a generic "structure prior" with no supervision, and a CGAN generated an object $x$ from condition $y$ and the latent representation learned by the unconditional GAN. Other papers explored applications of Conditional AAE models to the task of image modification (Antipov et al., 2017; Lample et al., 2017; Zhang et al., 2017).

CausalGAN (Kocaoglu et al., 2018) allowed components of the condition to have a dependency structure in the form of a causal model making conditions more complex. The Bayesian counterpart of AAE, the Variational Autoencoder (VAE) (Kingma and Welling, 2013), also had a conditional version (Sohn et al., 2015a), where conditions improved structured output prediction. CycleGAN (Zhu et al., 2017) examined a related task of object-to-object translation.

Multimodal learning models (Ngiam et al., 2011) and multiview representation models (Wang et al., 2016a) explored translations between different modalities, such as image to text. Wang et al. (2016b) presented a VAE-based generative multiview model. Our Bidirectional Adversarial Autoencoder provided explicit decoupling of latent representations and brought the multi-view approach into the AAE framework, where the basic Supervised AAE-like models (Makhzani et al., 2015) did not yield correct representations for sampling (Polykovskiy et al., 2018b).

Information decoupling ideas have been previously applied in other contexts: Yang et al. (2015) disentangled identity and pose factors of a 3D object; adversarial architecture from Mathieu et al. (2016) decoupled different factors in latent representations to transfer attributes between objects; Creswell et al. (2017) used VAE architecture with separate encoders for class label $y$ and latent representation $z$, forcing $z$ to exclude information about $y$; InfoVAE (Zhao et al., 2017) maximized mutual information between input and latent features; and Li et al. (2019) proposed a VAE modification that explicitly learns a "disentangled" representation $s$ to predict the class label and a "noninterpretable" representation $z$ that contains the rest of the information used for decoding.

InfoGAN (Chen X. et al., 2016) maximized mutual information between a subset of latent factors and the generator distribution. FusedGAN (Bodla et al., 2018) generated objects from two components, where only one component contains all object-relevant information. $\mathrm{Hu}$ et al. (2018) explicitly disentangles different factors in the latent representation and maps a part of the latent code to a particular external information.

\section{Conditional Generation for Biomedicine}

Machine learning has numerous applications in biomedicine and drug discovery (Gawehn et al., 2016; Mamoshina et al., 2016; Ching et al., 2018). Deep neural networks demonstrated positive results in various tasks, such as prediction of biological age (Putin et al., 2016; Mamoshina et al., 2018a; Mamoshina et al., 2019), prediction of targets and side effects Aliper et al., 2017; Mamoshina et al., 2018b; West et al., 2018), and applications in medicinal chemistry (Lusci et al., 2013; Ma et al., 2015).

Alongside large-scale studies that measure cellular processes, deep learning applications explore transcriptomics (Aliper et al., 2016b; Chen Y. et al., 2016); these works study cellular processes and their change following molecular perturbations. Deep learning has also been applied to pathway analysis (Ozerov et al., 2016), the prediction of protein functions (Liu, 2017), the discovery of RNA binding proteins (Zheng et al., 2017), the discovery of binding patterns of transcription factors (Qin and Feng, 2017), medical diagnostics based on omics data (Chaudhary et al., 2017), and the analysis of DNA and RNA sequences (Budach and Marsico, 2018).

In drug discovery, apart from predicting pharmacological properties and learning useful representations of small molecules (Duvenaud et al., 2015; Aliper et al., 2016a; Kuzminykh et al., 2018), deep learning is being widely applied to the generation of molecules (Sanchez and Aspuru-Guzik, 2018). Multiple authors have published models that generate new molecules that are similar to the training data or molecules with predefined properties (Kadurin et al., 2017a; Kadurin et al., 2017b; Segler et al., 2017 GómezBombarelli et al., 2018). AI-generated molecules have also been tested in vitro (Polykovskiy et al., 2018b). Reinforcement learning and generative models further enabled the generation of complex non-differentiable objectives, such as novelty (Guimaraes et al., 
2017; Putin et al., 2018a; Putin et al., 2018b). Generative models aim to eliminate the bottleneck of traditional drug development pipelines by providing promising new lead molecules for a specific target and automating the initial proposal of lead molecules with desired properties. Recently, Zhavoronkov et al. (2019) developed a model GENTRL to discover potent inhibitors of discoidin domain receptor 1 (DDR1) in 21 days.

\section{MODELS}

In this section, we introduce Unidirectional and a Bidirectional Adversarial Autoencoders and discuss their applications to conditional modeling. While we have focused on an example of molecular generation for transcriptome changes, in general, our model is not limited to these data types and can be used for generation in other domains.

\section{Supervised Adversarial Autoencoder}

Our model for conditional generation is based on a Supervised Adversarial Autoencoder (Supervised AAE, SAAE) (Makhzani et al., 2015) shown in Figure 1. The Supervised AAE learns three neural networks-an encoder $E_{x}$, a generator (decoder) $G_{x}$, and a discriminator $D$. The encoder maps a molecule $x$ onto a latent representation $z=E_{x}(x)$, and a generator reconstructs the molecule back from $z$ and gene expression changes $y: G_{x}(z, y)$.
We trained a discriminator $D$ to distinguish latent codes from samples of the prior distribution $p(z)$ and modified the encoder to make the discriminator believe that encoder's outputs are samples from the prior distribution:

$$
\begin{gathered}
\min _{E_{x}, G_{x}} \max _{D} \lambda_{1} \mathbb{E}_{\boldsymbol{x}, \boldsymbol{y} \sim p_{\mathrm{d}}(\boldsymbol{x}, \boldsymbol{y})} l_{\mathrm{rec}}^{\boldsymbol{x}}\left(\boldsymbol{x}, G_{\boldsymbol{x}}\left(E_{\boldsymbol{x}}(\boldsymbol{x}), \boldsymbol{y}\right)\right) \\
+\mathbb{E}_{z \sim p(\boldsymbol{z})} \log D(\boldsymbol{z})+\mathbb{E}_{\boldsymbol{x} \sim p_{\mathrm{d}}(\boldsymbol{x})} \log \left(1-D\left(E_{\boldsymbol{x}}(\boldsymbol{x})\right)\right),
\end{gathered}
$$

where $l_{\text {rec }}^{x}$ is a similarity measure between the original and reconstructed molecule, and $p_{\mathrm{d}}(x, y)$ is the data distribution. Hyperparameter $\lambda_{1}$ balances reconstruction and adversarial losses. We trained the model by alternately maximizing the loss in Equation 1 with respect to the parameters of $D$ and minimizing it with respect to the parameters of $E_{x}$ and $G_{x}$ (Goodfellow et al., 2014).

Besides passing gene expression changes $y$ directly to the generator, we could also train an autoencoder $\left(E_{y}, G_{y}\right)$ on $y$ and pass its latent codes to the molecular decoder $G_{x}$ (Figure 2). We call this model a Latent Supervised Adversarial Autoencoder (Latent SAAE). Its optimization problem is:

$$
\begin{gathered}
\min _{E_{x}, E_{y}, G_{x}, G_{y}} \max _{D} \lambda_{1} \mathbb{E}_{\boldsymbol{x}, \boldsymbol{y} \sim p_{\mathrm{d}}(\boldsymbol{x}, \boldsymbol{y})} l_{\mathrm{rec}}^{\boldsymbol{x}}\left(\boldsymbol{x}, G_{\boldsymbol{x}}\left(E_{\boldsymbol{x}}(\boldsymbol{x}), E_{\boldsymbol{y}}(\boldsymbol{y})\right)\right) \\
+\lambda_{2} \mathbb{E}_{\boldsymbol{y} \sim p_{\mathrm{d}}(\boldsymbol{y})} l_{\mathrm{rec}}^{y}\left(\boldsymbol{y}, G_{\boldsymbol{y}}\left(E_{\boldsymbol{x}}(\boldsymbol{y})\right)\right)+\mathbb{E}_{\boldsymbol{z} \sim p(\boldsymbol{z})} \log D(\boldsymbol{z}) \\
+\mathbb{E}_{\boldsymbol{x} \sim p_{\mathrm{d}}(\boldsymbol{x})} \log \left(1-D\left(E_{\boldsymbol{x}}(\boldsymbol{x})\right)\right) .
\end{gathered}
$$

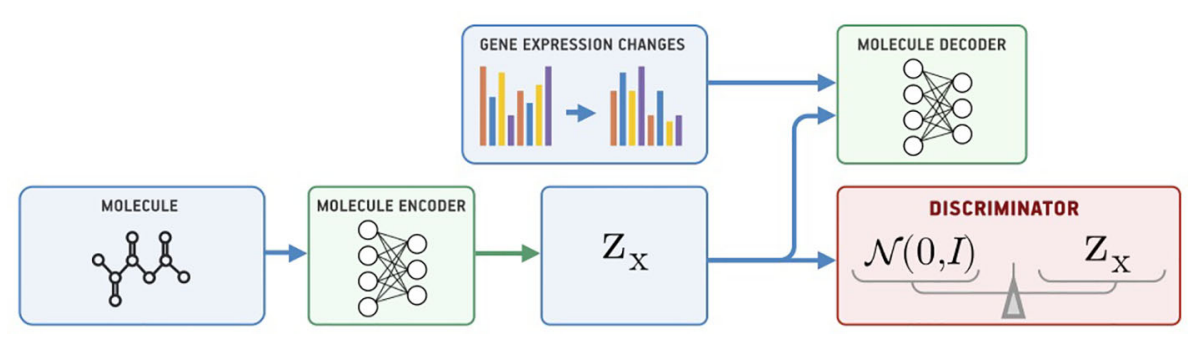

FIGURE 1 | The Supervised Adversarial Autoencoder model (SAAE).

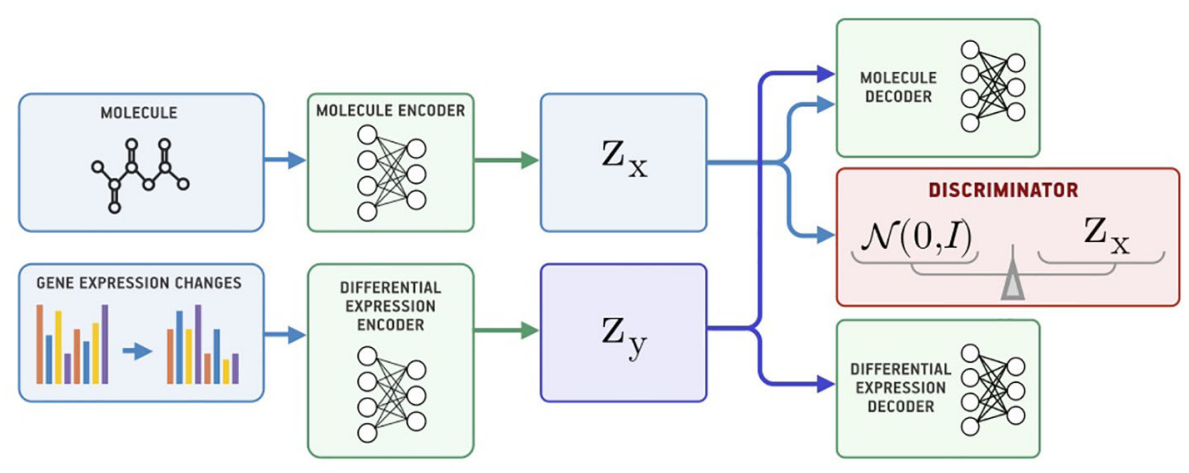

FIGURE 2 | The Latent Supervised Adversarial Autoencoder model (Latent SAAE). 
Hyperparameters $\lambda_{1}$ and $\lambda_{2}$ balance object and condition reconstruction losses as well as the adversarial loss.

\section{Bidirectional Adversarial Autoencoder}

Both SAAE and Latent SAAE models learn conditional distribution $p(x \mid y)$ of molecules for specific transcriptome changes. In this paper, we learned a joint distribution $p(x, y)$ instead. Our model is symmetric in that it can generate both $x$ for a given $y$ and $y$ for a given $x$. We assume that the data are generated with a graphical model shown in Figure 3. Latent variables $z_{x}$ and $z_{y}$ are exclusive parts that represent features specific only to molecules or transcriptome changes. Latent variable $s$ represents a shared part that describes features significant for both molecules and expression changes. To produce a new data point, we sampled exclusive $\left(z_{x}, z_{y}\right)$ and shared $(s)$ parts independently and used generative distributions $G_{x}\left(x \mid s, z_{x}\right)$ and $G_{y}\left(y \mid s, z_{y}\right)$ to produce $x$ and $y$.

To train a model, we used inference networks that predict values of $s, z_{x}$, and $z_{y}: E_{x}\left(z_{x} \mid x\right), E_{y}\left(z_{y} \mid y\right)$, and $E(s \mid x, y)=E_{x}(s \mid x)$ $=E_{y}(s \mid y)$. Note that we used two separate networks for inference of $s$ from one of $x$ and $y$ to perform conditional sampling (when only one of $x$ or $y$ is known). For example, to sample $p(x \mid y)$, we would do the following steps:

$$
\boldsymbol{s} \sim E_{y}(\boldsymbol{s} \mid \boldsymbol{y}), \quad z_{x} \sim p\left(z_{x}\right), \quad x \sim G_{x}\left(s, z_{x}\right) .
$$

For the molecule, $s$ may describe its pharmacophorebinding points that are recognized by macromolecules. For the gene expression, $s$ may describe affected proteins. Note that we can infer pharmacophore from a list of affected genes and vice versa. The exclusive part $z_{x}$ of a molecule describes the remaining structural parts besides the pharmacophore points. The exclusive part $z_{y}$ of a transcriptome describes cellular processes that influence the expression but are not caused by the drug.

Figure 4 shows the proposed Bidirectional AAE architecture. We used two deterministic encoders $E_{x}$ and $E_{y}$ that infer latent codes from molecules and transcriptomes:

$$
\left(z_{x}, s_{x}\right)=E_{x}(x), \quad\left(z_{y}, s_{y}\right)=E_{y}(y)
$$

Two deterministic decoders (generators) $G_{x}$ and $G_{y}$ reconstruct molecules $x$ and gene expression changes $y$ back from the latent codes:

$$
x=G_{x}\left(z_{x}, s_{x}\right), \quad y=G_{y}\left(z_{y}, s_{y}\right)
$$

The objective function consists of three parts, each capturing restrictions from the graphical model-the structure of the shared representation, reconstruction quality, and independence of shared and exclusive representations.

Shared loss ensures that shared representations extracted from the molecule $s_{x}$ and gene expression $s_{y}$ are close to each other, as suggested by the graphical model:

$$
\min _{E_{x}, E_{y}} \mathcal{L}_{\text {shared }}=\mathbb{E}_{\boldsymbol{x}, \boldsymbol{y} \sim P \mathrm{~d}(\boldsymbol{x}, \boldsymbol{y})}\left\|\boldsymbol{s}_{\boldsymbol{x}}-\boldsymbol{s}_{\boldsymbol{y}}\right\|_{2}^{2} .
$$

Reconstruction loss ensures that decoders reconstruct molecules and gene expressions back from the latent codes produced by the encoders. We also use a cross-reconstruction loss, where molecular decoder $E_{x}$ uses shared part $s_{y}$ from a gene expression encoder $E_{y}$ for reconstruction and vice versa:

$$
\begin{aligned}
\min _{E_{x}, E_{y}, G_{x}} \mathcal{L}_{\mathrm{rec}}^{x}= & \mathbb{E}_{\boldsymbol{x} \sim p_{\mathrm{d}}(\boldsymbol{x})} l_{\mathrm{rec}}^{x}\left(\boldsymbol{x}, G_{\boldsymbol{x}}\left(\boldsymbol{z}_{\boldsymbol{x}}, \boldsymbol{s}_{\boldsymbol{x}}\right)\right) \\
& +\mathbb{E}_{\boldsymbol{x}, \boldsymbol{y} \sim p_{\mathrm{d}}(\boldsymbol{x}, \boldsymbol{y})} \text { lec }_{\mathrm{rec}}^{x}\left(\boldsymbol{x}, G_{\boldsymbol{x}}\left(\boldsymbol{z}_{\boldsymbol{x}}, \boldsymbol{s}_{\boldsymbol{y}}\right)\right) \\
\min _{E_{x}, E_{y}, G_{y}} \mathcal{L}_{\mathrm{rec}}^{y}= & \mathbb{E}_{\boldsymbol{y} \sim p_{\mathrm{d}}(\boldsymbol{y})} l_{\mathrm{rec}}^{y}\left(\boldsymbol{y}, G_{\boldsymbol{x}}\left(\boldsymbol{z}_{\boldsymbol{y}}, \boldsymbol{s}_{\boldsymbol{y}}\right)\right) \\
& +\mathbb{E}_{\boldsymbol{x}, \boldsymbol{y} \sim p_{\mathrm{d}}(\boldsymbol{x}, \boldsymbol{y})} l_{\mathrm{rec}}^{y_{\mathrm{rac}}}\left(\boldsymbol{y}, G_{\boldsymbol{y}}\left(\boldsymbol{z}_{\boldsymbol{x}}, \boldsymbol{s}_{\boldsymbol{y}}\right)\right)
\end{aligned}
$$

where $l_{\text {rec }}^{x}$ and $l_{\mathrm{rec}}^{y}$ are some distance measures in the molecules and gene expression space.

Discriminator loss is an objective that encourages distributions $p(s), p\left(z_{x}\right)$, and $p\left(z_{y}\right)$ to be independent, which means that shared and exclusive parts must learn different features. This restriction comes from a graphical model. It also encourages $p(s), p\left(z_{x}\right)$, and $p\left(z_{y}\right)$ to be standard Gaussian distributions $N(0, I)$ to perform a sampling scheme from Equation 3. We optimized the discriminator in an adversarial manner (Goodfellow et al., 2014) similar to SAAE:

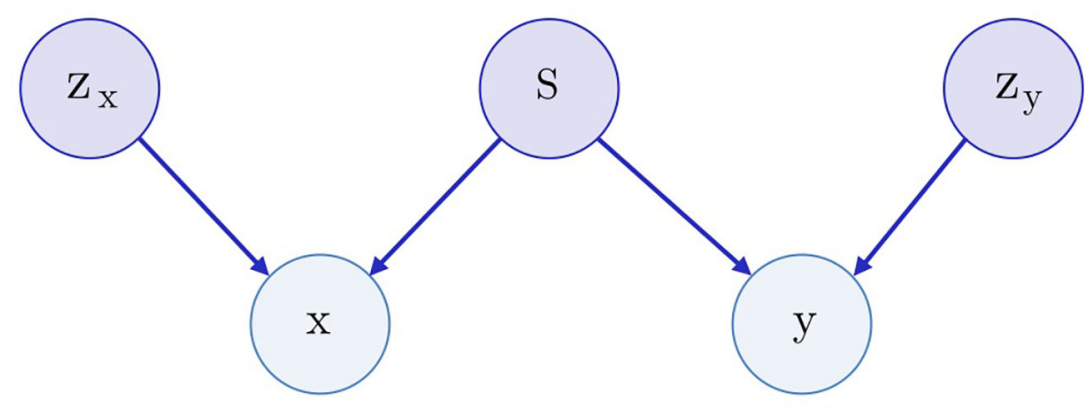

FIGURE 3 | The underlying graphical model of the data: molecules $x$, gene expression changes $y$, three latent variables correspond to the exclusive $\left(z_{x}, z_{y}\right)$ and shared (s) features between $x$ and $y$. 


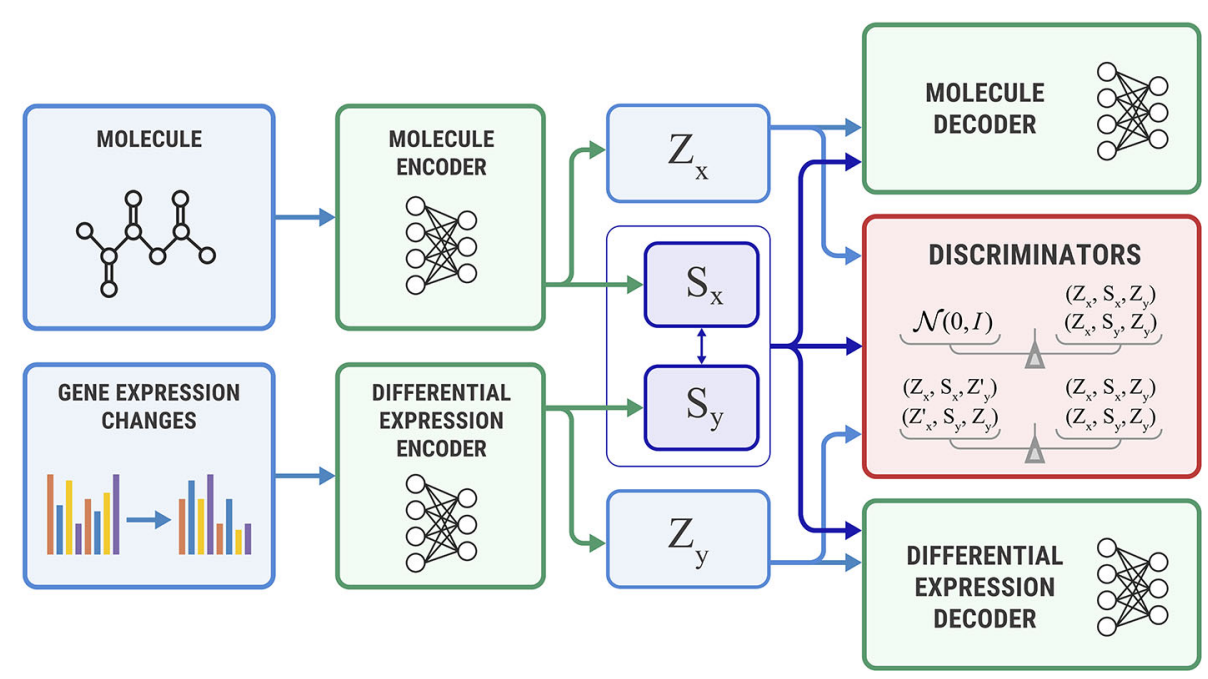

FIGURE 4 | The Bidirectional Adversarial Autoencoders model. The discriminators ensure that three latent code components are independent and indistinguishable from the prior distribution.

$$
\begin{aligned}
\min _{E_{x}, E_{y}, G_{x}, G_{y}} \max _{D} \mathcal{L}_{\mathrm{adv}}=\mathbb{E}_{\boldsymbol{s}^{\prime}, \boldsymbol{z}_{x}^{\prime}, \boldsymbol{z}_{y}^{\prime} \sim p(\boldsymbol{s}) p\left(\boldsymbol{z}_{\boldsymbol{x}}\right) p\left(\boldsymbol{z}_{y}\right)} \log D\left(\boldsymbol{z}_{\boldsymbol{x}}^{\prime}, \boldsymbol{s}^{\prime}, \boldsymbol{z}_{y}^{\prime}\right) \\
+\frac{1}{2} \mathbb{E}_{\boldsymbol{x}, \boldsymbol{y} \sim p_{\mathrm{d}}(\boldsymbol{x}, \boldsymbol{y})} \log \left(1-D\left(\boldsymbol{z}_{\boldsymbol{x}}, \boldsymbol{s}_{\boldsymbol{x}}, \boldsymbol{z}_{\boldsymbol{y}}\right)\right) \\
+\frac{1}{2} \mathbb{E}_{\boldsymbol{x}, \boldsymbol{y} \sim p_{\mathrm{d}}(\boldsymbol{x}, \boldsymbol{y})} \log \left(1-D\left(\boldsymbol{z}_{\boldsymbol{x}}, \boldsymbol{s}_{\boldsymbol{y}}, \boldsymbol{z}_{\boldsymbol{y}}\right)\right)
\end{aligned}
$$

Note that since the target distribution for adversarial training is factorized, we expected that the trained model would learn independence of $s, z_{x}$, and $z_{y}$.

Additional discriminator losses We also added additional discrimination objective to explicitly encourage independence of $z_{x}$ from $\left(s_{y}, z_{y}\right)$ and $z_{y}$ from $\left(s_{x}, z_{x}\right)$ :

$$
\begin{gathered}
\min _{E_{x}, E_{y}, G_{x}, G_{y}} \max _{D} \mathcal{L}_{\text {info }}=\mathbb{E}_{\boldsymbol{x}, \boldsymbol{y} \sim p_{\mathrm{d}}(\boldsymbol{x}, \boldsymbol{y})} \mathbb{E}_{\boldsymbol{y}^{\prime} \sim p_{\mathrm{d}}(\boldsymbol{y})} \\
{\left[\log D\left(\boldsymbol{z}_{\boldsymbol{x}}, \boldsymbol{s}_{\boldsymbol{x}}, \boldsymbol{z}_{\boldsymbol{y}}\right)+\log \left(1-D\left(\boldsymbol{z}_{\boldsymbol{x}}, \boldsymbol{s}_{\boldsymbol{x}}, \boldsymbol{z}_{\boldsymbol{y}}^{\prime}\right)\right)\right]} \\
+\mathbb{E}_{\boldsymbol{x}, \boldsymbol{y} \sim p_{\mathrm{d}}(\boldsymbol{x}, \boldsymbol{y})} \mathbb{E}_{\boldsymbol{x}^{\prime} \sim p_{\mathrm{d}}(\boldsymbol{x})}\left[\log D\left(\boldsymbol{z}_{\boldsymbol{x}}, \boldsymbol{s}_{\boldsymbol{y}}, \boldsymbol{z}_{\boldsymbol{y}}\right)+\log \left(1-D\left(\boldsymbol{z}_{\boldsymbol{x}}^{\prime}, \boldsymbol{s}_{\boldsymbol{y}}, \boldsymbol{z}_{\boldsymbol{y}}\right)\right)\right],
\end{gathered}
$$

where $z_{x}^{\prime}$ is an exclusive latent code of $x^{\prime}$, and $z_{y}^{\prime}$ is an exclusive latent code of $y^{\prime}$. In practice, we obtain $z_{x}{ }^{\prime}$ and $z_{y}{ }^{\prime}$ by shuffling $z_{x}$ and $z_{y}$ in each batch.

Combining these objectives, the final optimization problem becomes a minimax problem that can be solved by alternating gradient descent with respect to encoder and decoder parameters, and gradient ascent with respect to the discriminator parameters:

$$
\min _{E_{x}, E_{y}, G_{x}, G_{y}} \max _{D} \lambda_{1} \mathcal{L}_{\text {shared }}+\lambda_{2} \mathcal{L}_{\text {rec }}^{x}+\lambda_{3} \mathcal{L}_{\text {rec }}^{y}+\mathcal{L}_{\text {adv }}+\mathcal{L}_{\text {info }} .
$$

The hyperparameters $\lambda_{1}, \lambda_{2}$, and $\lambda_{3}$ balance different objectives. In general, we optimize lambdas based on the performance of BiAAE on the holdout set in terms of the target metrics, such as estimated negative conditional loglikelihood. In practice, we found that optimal values of lambdas yielded the gradients of loss components on a similar scale.

\section{Unidirectional Adversarial Autoencoder}

The Bidirectional AAE can generate molecules that cause given transcriptome changes and transcriptome changes caused by a given molecule. However, if we only need conditional generation of molecules $p(x \mid y)$, we simplify the model by removing the encoder of $s_{x}$. The encoder $E_{x}$ returns only an exclusive part: $z_{x}=$ $E_{x}(x)$. For this model, we derived the objective from Equation 11 by setting $s_{x}$ equal to $s_{y}$ (Figure 5).

\section{EXPERIMENTAL EVALUATION}

In this section, we have described the experimental setup and presented numerical results on the toy Noisy MNIST dataset and a LINCS L1000 dataset (Duan et al., 2014) of gene expression data.

\section{Noisy MNIST}

We start by validating our models on the Noisy MNIST (Wang et al., 2015) dataset of image pairs $(x, y)$, for which we know the correct features in the shared representation $s$. The image $x$ is a handwritten digit randomly rotated by an angle in $[-\pi / 4, \pi / 4]$. The image $y$ is also a randomly rotated version of another image containing the same digit as $x$ but with strong additive Gaussian noise. As a result, the only common feature between $x$ and $y$ is the digit. Bidirectional and Unidirectional AAEs should learn to store only the information about the digit in $s$.

The train-validation-test splits contain 50,000, 10,000, and 10,000 samples respectively. We set the batch size to 128 and the learning rate to 0.0003 , and we used the Adam (Kingma and $\mathrm{Ba}, 2015)$ optimizer with $\beta_{1}=0.5, \beta_{2}=0.9$ for models with 


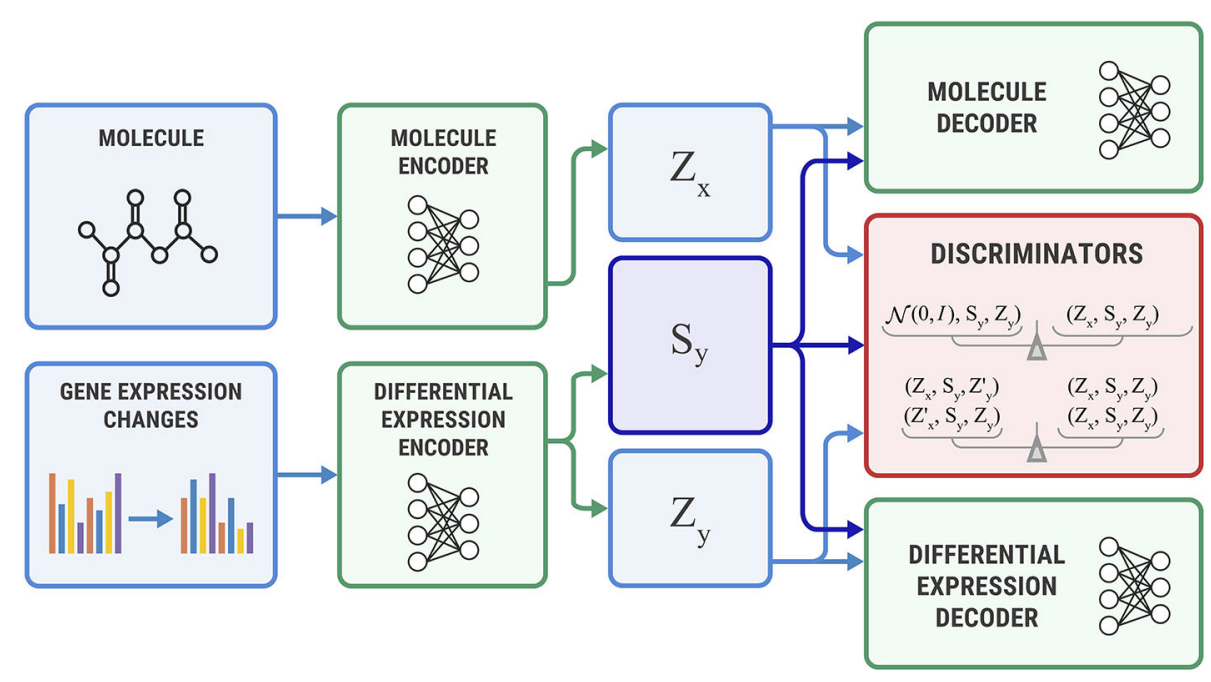

FIGURE 5 | The Unidirectional Adversarial Autoencoder: a simplified version of a Bidirectional Adversarial Autoencoder for generating from $\boldsymbol{p}(\boldsymbol{x} \mid \boldsymbol{y})$. The discriminator part ensures that the three latent code components are independent, and the object's exclusive latent code is indistinguishable from the prior distribution.

adversarial training and $\beta_{1}=0.99$ and $\beta_{1}=0.999$ for others with a single update of autoencoders per a single update of the discriminator. Encoder and decoder architectures were the same for all models, with 12-dimensional $z_{x}, z_{y}$ and 4-dimensional $s$. The encoder had 2 convolutional layers with a number of channels $1 \rightarrow 32 \rightarrow 16$ with $2 \mathrm{D}$ dropout rate 0.2 followed by three fullyconnected layers of size $64 \rightarrow 128 \rightarrow 128 \rightarrow 16$ with batch normalization. The decoder consisted of 2 fully connected layers followed by 3 transposed convolution layers; the discriminators have two hidden layers with $1024 \rightarrow 512$ units. We set the weights for $\mathcal{L}_{\text {rec }}$ to 10 and 0.1 for $\mathcal{L}_{\text {shared. }}$ Other $\lambda$ were set to 1 . For Unidirectional AAE, we increased weight for $\mathcal{L}_{\text {info }}$ to 100 . For baseline models we used similar architectures. Please refer to the Supplementary Material for additional hyperparameters.

Conditional generative model $p(\boldsymbol{x} \mid \boldsymbol{y})$ should produce images with the same digit as image $y$, which we evaluate by training a separate convolutional neural network to predict the digit from $x$ and comparing the most probable digit to the actual digit of $y$ known from the dataset. We also estimated a conditional mutual information $\mathcal{M I}\left(\boldsymbol{x}, \boldsymbol{s}_{\boldsymbol{y}} \mid \boldsymbol{y}\right)$ using a Mutual Information Neural Estimation (MINE) (Belghazi et al., 2018) algorithm for BiAAE, UniAAE, JMVAE, and VCCA models. For SAAE, LatentSAAE, CVAE, and VIB we estimated $\mathcal{M I}(\boldsymbol{x}$, $\boldsymbol{s} \mid \boldsymbol{y})$ since these models do not separate embeddings into shared and exclusive parts explicitly. Models with high mutual information extract relevant information from $y$. A neural network for MINE consisted of a convolutional encoder for $x$ and fully-connected encoder for $s_{y}$. We then passed a concatenated embedding through a fully-connected neural network to get a final estimate of mutual information. Results in Table 1 suggest that the BiAAE model extracted relevant mutual information which, besides all, contained information about the digit of $y$. In Figure 6, we show example samples from the model.

\section{Differential Gene Expression}

In this section, we have validated Bidirectional AAE on a gene expression profiles dataset with 978 genes. We use a dataset of transcriptomes from the Library of Integrated Network-based Cellular Signatures (LINCS) L1000 project (Duan et al., 2014). The database contains measurements of gene expressions before and after cells react with a molecule at a given concentration.

For each cell line, the training set contains experiments characterized by the control $\left(\mathrm{ge}_{b} \in \mathbb{R}^{978}\right)$ and perturbationinduced $\left(\mathrm{ge}_{a} \in \mathbb{R}^{978}\right.$ ) gene expression profiles. We represented molecular structures in the SMILES format (Weininger, 1988; Weininger et al., 1989). We augmented the dataset by randomly matching control and perturbation-induced measurements from the same plate.

We preprocessed the training dataset by removing molecules with a molecular weight less than 250 and more than $550 \mathrm{Da}$. We then removed molecules that did not contain any oxygen or nitrogen atoms or contained atoms besides $\mathrm{C}, \mathrm{N}, \mathrm{S}, \mathrm{O}, \mathrm{F}, \mathrm{Cl}, \mathrm{Br}$, and $\mathrm{H}$. Finally, we removed molecules that contained rings with

TABLE 1 | Quantitative results for a Noisy MNIST experiment. Conditional Generation section evaluates how often the model produced a correct digit. Latent Codes section estimates the Mutual Information between $z_{x}$ and $s$ ( $y$ for SAAE).

\begin{tabular}{lccc}
\hline Model & Accuracy, $\%$ & MI(x,s $\mathbf{y} \mid \boldsymbol{y})$ & MI(x,s|y) \\
\hline SAAE (Makhzani et al., 2015) & 43.68 & - & 1.665 \\
Latent SAAE & 34.76 & - & $\mathbf{1 . 6 8 1}$ \\
CVAE (Sohn et al., 2015b) & 0.4583 & - & 0.3074 \\
JMVAE (Suzuki et al., 2017) & 5.38 & 0.9515 & - \\
VIB (Alemi et al., 2017) & 43.6 & - & 1.121 \\
VCCA (Wang et al., 2016b) & 23.35 & 1.239 & - \\
BiAAE (our) & $\mathbf{4 9 . 2 1}$ & 1.432 & - \\
UniAAE (our) & 47.61 & $\mathbf{1 . 6 2 7}$ & -
\end{tabular}




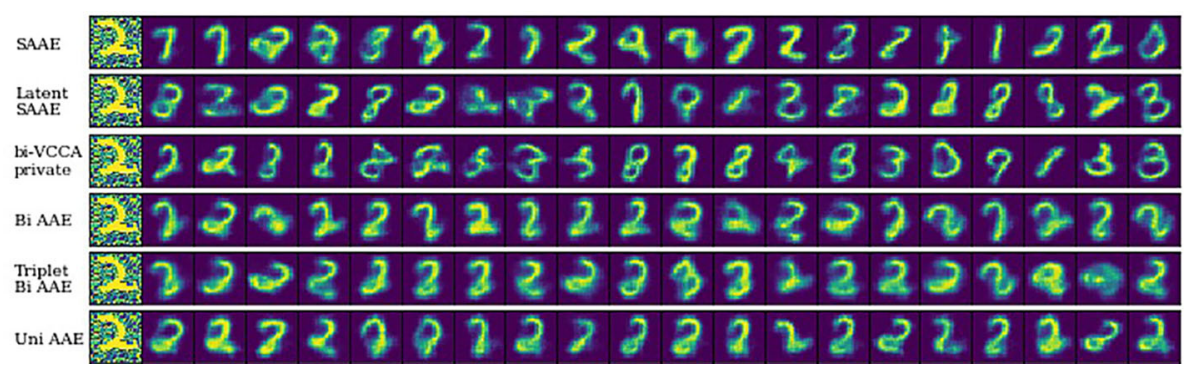

FIGURE 6 | Qualitative results on a Noisy MNIST dataset. The figure shows generated images $x$ for a noisy image $y$ (left column) as a condition. Generated images must have the same digit as $y$.

more than eight atoms or tetracyclines. The resulting dataset contained 5,216 unique SMILES. Since the dataset is small, we pretrained an autoencoder on the MOSES (Polykovskiy et al., 2018a) dataset and used its encoder and decoder as initial weights in all models.

For all baseline models on differential gene expressions, we used similar hyperparameters shown in Table 2 (please refer to the Supplementary Material for the exact hyperparameters). In all experiments, we split our dataset into train, validation, and test sets, all containing different drugs. To construct a training example, we sampled a drug-dose pair, a perturbation for this drug and dose, and a control expression from the same plate as the perturbed expression.

We used a two-step encoder for $y=(\eta, \Delta$ ge $)$ shown in Figure 7 , where $\Delta \mathrm{ge}=\mathrm{ge}_{a}-\mathrm{ge}_{b}$. We first embedded $\Delta \mathrm{ge}$ with a fullyconnected neural network, and then concatenated the obtained representation with a logarithm of concentration $\eta$. We passed the resulting vector through a final encoder. The decoder has a symmetric architecture.

\section{Generating Molecular Structures for Gene Expression Profiles}

The proposed BiAAE model can generate molecules for given gene expression changes and vice versa. We started by experimenting with the molecular generation (Table 3). In the experiment, we reported a negative log-probability of generating the exact incubated drug $x$ given the dose and gene expression change averaged over tokens $\log p(x \mid \Delta$ ge, $\eta)$. We also estimated a Mutual Information $\mathcal{M I}\left(\boldsymbol{x}, s_{y} \mid \Delta\right.$ ge, $\left.\eta\right)$ similar to the MNIST experiment described above. For each $\eta$ and $\Delta$ ge, we generated a set of molecules $G$ and estimated a fraction of valid molecules and internal diversity of $G$ :

$$
\begin{gathered}
\operatorname{IntDiv}(G)=1-\frac{1}{|G|(|G|-1)} \sum_{\substack{m_{1}, m_{2} \in G \\
m_{1} \neq m_{2}}} T\left(m_{1}, m_{2}\right), \\
\end{gathered}
$$

where $T$ is a Tanimoto similarity on Morgan fingerprints. This metric shows whether a model can produce multiple candidates for a given gene expression or collapses to a single molecule.

TABLE 2 | Hyperparameters for neural networks training on gene expression data. All neural networks are fully connected, and decoders have an architecture symmetric to the encoders.

\begin{tabular}{lc}
\hline Hyperparameter & Value \\
\hline Molecular Encoder & GRU; hidden size 128; 2 layers \\
Expression Encoder & $\mathrm{IN}(978) \rightarrow 256 \rightarrow$ OUT $(128)$ \\
Difference Encoder & $\mathrm{IN}(129) \rightarrow 128 \rightarrow$ OUT $(10+10)$ \\
Discriminator & $\mathrm{IN} \rightarrow 1024 \rightarrow 512 \rightarrow$ OUT(1) \\
Batch Normalization & After each linear layer in encoders \\
Activation Function & LeakyReLU \\
Learning Rate & 0.0003
\end{tabular}

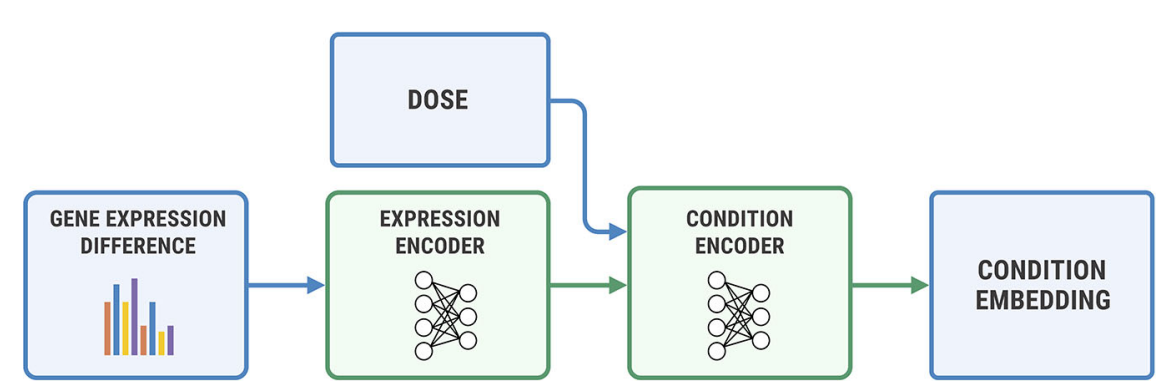

FIGURE 7 | The architecture of the condition encoder for changes in the transcriptome. The input to the expression encoder is the difference between the control and perturbed expressions. We passed the dose to the last layers of the encoder. 
The proposed BiAAE and UniAAE architectures show the ability to capture the dependencies in the training set and generalize to new objects from the validation set. The BiAAE model provides better mutual information while preserving valid diverse molecules.

\section{Comparing Generated Molecular Structures to Known Active Molecules}

In this experiment, we show that the proposed generative model (BiAAE) can produce biologically meaningful results. We used a manually curated database of bioactive molecules ChEMBL 24.1 (Gaulton et al., 2016) and additional profiles of gene expression knockdown from LINCS L1000 (Duan et al., 2014).

The first experiment evaluates molecular generation given a transcriptome change of a small molecule inhibitor of a specific protein. The ChEMBL dataset has experimental data on molecules that inhibit a certain human protein. We chose template molecules that are present in both LINCS molecule perturbation dataset and ChEMBL dataset. We used molecules that had inhibition concentration less than $10 \mu \mathrm{M}$ IC50 for only one protein.

The condition for molecular generation is a transcriptome change and a dose of a template molecule. Specifically, the condition is a shared part $s_{y}$ of the gene expression and dose embedding. The model is expected to generate molecules that are similar to known drugs. In Figure 8, for several protein targets,

TABLE 3 | Validation results of conditional generation $p(x \mid \Delta$ ge, $\eta)$.

\begin{tabular}{lccccc}
\hline Model & NLL & $\begin{array}{c}\text { MI } \\
\left(\boldsymbol{x}, \mathbf{s}_{\mathbf{y}} \mid \Delta \mathbf{g e}, \boldsymbol{\eta}\right)\end{array}$ & $\begin{array}{c}\mathbf{M I} \\
(\boldsymbol{x}, \mathbf{s} \mid \Delta \mathbf{g e}, \boldsymbol{\eta})\end{array}$ & $\begin{array}{c}\text { Internal } \\
\text { Diversity }\end{array}$ & Validity \\
\hline SAAE & 0.55 & - & $\mathbf{0 . 1 1}$ & $\mathbf{0 . 8 5}$ & 0.64 \\
Latent SAAE & 0.55 & - & 0.00 & $\mathbf{0 . 8 5}$ & 0.62 \\
CVAE & 1.22 & - & 0.00 & 0.84 & 0.58 \\
JMVAE & 1.42 & 0.00 & - & 0.61 & $\mathbf{0 . 8 2}$ \\
VIB & 1.46 & - & 0.00 & 0.17 & 0.29 \\
VCCA & 1.36 & 0.00 & - & 0.53 & 0.71 \\
BiAAE & 0.77 & $\mathbf{0 . 3 2}$ & - & $\mathbf{0 . 8 5}$ & 0.76 \\
UniAAE & $\mathbf{0 . 5 3}$ & 0.00 & - & $\mathbf{0 . 8 5}$ & 0.61
\end{tabular}

we show a known inhibitor and generated molecules that could induce similar transcriptome profile changes.

The second experiment evaluates molecular generation given a transcriptome change of a specific gene knockdown. The LINCS dataset contains gene knockdown transcriptomes that the model was not trained on. For each gene knockdown, we found a corresponding human protein in the ChEMBL dataset. We chose template molecules that had a proven IC50 less than $10 \mu \mathrm{M}$ for only one protein. The condition for molecular generation is a transcriptome change of a gene knockdown and the most common dose $10 \mu \mathrm{M}$ in LINCS. The model is expected to generate molecules that produce the same transcriptome change of gene knockdowns.

The condition is different compared to the previous experiment in a way that the gene knockdown expression profile is not induced by a small molecule but rather shows the desired behavior of the potential drug. In Figure 9, we show generated molecules and compare them to known inhibitors of a protein corresponding to a knocked down gene. We expect these molecules to produce similar effects in gene expression to gene knockdown.

\section{Predicting Gene Expression Profiles for an Incubated Drug}

We experimented with predicting gene expression changes after drug incubation (Table 4). First, we report estimated mutual information $\mathcal{M I}\left(\Delta\right.$ ge, $\left.\eta, \boldsymbol{s}_{\boldsymbol{x}} \mid \boldsymbol{x}\right)$ similar to the previous experiments. We also report the $R^{2}$ metric, which measures the determination coefficient between the real and predicted $(\Delta$ ge, $\eta$ ) for a given molecule. Finally, we report a top-1 precision metric, which shows the fraction of samples for which the largest absolute change in real and predicted $\Delta$ ge matched.

To compute $R^{2}$ and top- 1 precision, we only used drugs that were administered at $\eta=10 \mu \mathrm{M}$ concentration. Since we are only interested in a certain concentration, we discarded generated ( $\Delta$ ge, $\eta$ ) tuples if $\eta$ was far from $10 \mu \mathrm{M}$ (outside the range [-6.5, $-5.5]$ in $\log _{10}$ scale). Note that VIB was not able to generate any gene expression changes near $10 \mu \mathrm{M}$.

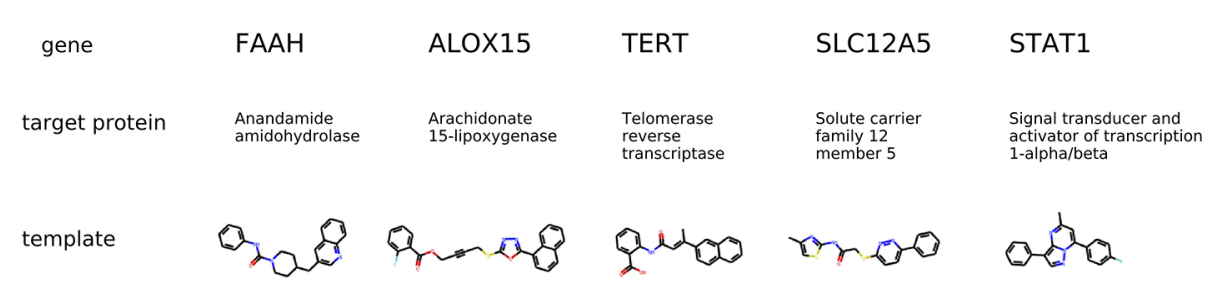

generated

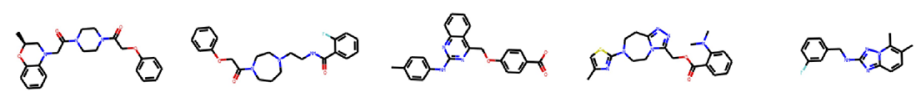

FIGURE 8 | The examples of generated molecules conditioned on gene expression changes from a protein inhibitor; Real most similar inhibitors from ChEMBL are provided for comparison. 


\begin{tabular}{|c|c|c|c|c|c|c|}
\hline gene & MAPK14 & FAAH & MELK & NAMPT & NAMPT & AKR1C3 \\
\hline target protein & $\begin{array}{l}\text { MAP kinase } \\
\text { p38 alpha }\end{array}$ & $\begin{array}{l}\text { Anandamide } \\
\text { amidohydrolase }\end{array}$ & $\begin{array}{l}\text { Maternal embryonic } \\
\text { leucine zipper } \\
\text { kinase }\end{array}$ & $\begin{array}{l}\text { Nicotinamide } \\
\text { phosphoribo- } \\
\text { syltransferase }\end{array}$ & $\begin{array}{l}\text { Nicotinamide } \\
\text { phosphoribo- } \\
\text { syltransferase }\end{array}$ & $\begin{array}{l}\text { Aldo-keto-reductase } \\
\text { family } 1 \\
\text { member } \mathrm{C} 3\end{array}$ \\
\hline template & & & & & & Q.tob \\
\hline generated & & & & & & bong \\
\hline
\end{tabular}

FIGURE 9 | The examples of generated molecules conditioned on gene expression changes from a gene knockdown; Real most similar inhibitors of a knocked down gene are provided for comparison.

TABLE 4 | Validation results of conditional generation $\boldsymbol{p}(\Delta \boldsymbol{g e}, \boldsymbol{\eta} \mid \boldsymbol{x})$.

\begin{tabular}{lcccc}
\hline Model & $\mathbf{M I}\left(\boldsymbol{\Delta} \mathbf{g e}, \boldsymbol{\eta}, \mathbf{s}_{\mathbf{y}} \mid \mathbf{x}\right)$ & $\mathbf{M I}(\boldsymbol{\Delta} \mathbf{g e}, \boldsymbol{\eta}, \mathbf{s} \mid \mathbf{x})$ & Top-1 precision & $\boldsymbol{R}^{\mathbf{2}}$ score \\
\hline SAAE & - & 0.00 & 0.58 & 0.26 \\
Latent SAAE & - & $\mathbf{0 . 2 3}$ & 0.74 & 0.28 \\
CVAE & - & 0.01 & 0.29 & $\mathbf{0 . 3 3}$ \\
JMVAE & 0.00 & - & 0.0 & 0.03 \\
VIB & - & 0.00 & - & - \\
VCCA & 0.00 & - & 0.0 & 0.03 \\
BiAAE & 0.20 & - & 0.74 & 0.32 \\
UniAAE & $\mathbf{0 . 2 1}$ & - & $\mathbf{0 . 7 7}$ & 0.27 \\
\hline
\end{tabular}

The experiment demonstrates that proposed UniAAE, BiAAE, and LatentSAAE models generalize well the symmetric task and show good metrics on predicting gene expression changes.

\section{DISCUSSION}

The key advantage of the proposed model compared to the previous works is the joint adversarial learning of latent representations of paired objects. This representation improves conditional generation metrics and shows promising results in molecular generation for desired transcriptome changes.

Three discriminator neural networks ensure that the latent representations divided into shared and exclusive parts are more meaningful and useful for the conditional generation. Two additional discriminator losses help the model learn a more expressive shared part and make sure that all three parts are mutually independent.

However, adversarial training slightly complicates the training procedure for the BiAAE model. In comparison with other baseline models, the training loss contains more terms, each with a coefficient to tune. In general, we tune these coefficients using grid search, and we select the best coefficients according to the generative metrics on the validation set. In practice, we simplify the grid search and use the same coefficient for the adversarial terms $\lambda_{1}=\lambda_{4}=\lambda_{5}$ since the corresponding losses have values on the same scale. We choose the search space for coefficients $\lambda_{2}, \lambda_{3}$ in a way that the second and third terms provide the gradient in the same scale as the other terms.

Another problem that arises when we use the adversarial approach is the instability of training. The instability is the consequence of the minimax nature of adversarial training (Goodfellow et al., 2014). To overcome the instability, we use approaches described in (Bang and Shim, 2018), i.e., we use shallow discriminators and Adam optimizer with parameters $\beta_{1}=0.5, \beta_{2}=0.9$.

\section{CONCLUSION}

In this work, we proposed a Bidirectional Adversarial Autoencoder model for the generation of molecular structures for given gene expression changes. Our AAE-based architecture extracts shared information between molecule and gene expression changes and separates it from the remaining exclusive information. We showed that our model outperforms baseline conditional generative models on the Noisy MNIST dataset and the generation of molecular structures for the desired transcriptome changes.

\section{DATA AVAILABILITY STATEMENT}

The code and datasets for this study are available at https:// github.com/insilicomedicine/BiAAE.

\section{AUTHOR CONTRIBUTIONS}

RS and MK implemented the BiAAE and baseline models and conducted the experiments. RS, AK, and AA prepared the datasets. RS, MK, AK, and DP derived the BiAAE and 
UniAAE models. RS, AZ, AK, SN, and DP wrote the manuscript. $\mathrm{AK}$ and DP supervised the project.

\section{ACKNOWLEDGMENTS}

The original idea for molecular generation for a specific transcriptional or proteomic profile, a technology used broadly at Insilico Medicine, was proposed in 2016 by Dr. Alex

\section{REFERENCES}

Alemi, A. A., Fischer, I., Dillon, J. V., and Murphy, K. (2017). Deep Variational Information Bottleneck. Int. Conf. Learn. Representations.

Aliper, A., Plis, S., Artemov, A., Ulloa, A., Mamoshina, P., and Zhavoronkov, A. (2016a). Deep Learning Applications for Predicting Pharmacological Properties of Drugs and Drug Repurposing Using Transcriptomic Data. Mol. Pharm. 13, 2524-2530. doi: 10.1021/acs.molpharmaceut.6b00248

Aliper, A. M., Plis, S. M., Artemov, A. V., Ulloa, A., Mamoshina, P., and Zhavoronkov, A. (2016b). Deep learning applications for predicting pharmacological properties of drugs and drug repurposing using transcriptomic data. Mol. Pharm. 13 7,25242530. doi: 10.1021/acs.molpharmaceut.6b00248

Aliper, A., Jellen, L., Cortese, F., Artemov, A., Karpinsky-Semper, D., Moskalev, A., et al. (2017). Towards Natural Mimetics of Metformin and Rapamycin. Aging (Albany NY) 9, 2245-2268. doi: 10.18632/aging.101319

Antipov, G., Baccouche, M., and Dugelay, J. (2017). Face aging with conditional generative adversarial networks. In. 2017 IEEE Int. Conf. Image Process. (ICIP)., 2089-2093. doi: 10.1109/ICIP.2017.8296650

Bang, D., and Shim, H. (2018). "Improved training of generative adversarial networks using representative features," in Proceedings of the 35th International Conference on Machine Learning, vol. 80. Eds. J. Dy and A. Krause (Stockholmsmässan, Stockholm Sweden: PMLR), 433-442.

Belghazi, M. I., Baratin, A., Rajeshwar, S., Ozair, S., Bengio, Y., Hjelm, D., et al. (2018). "Mutual information neural estimation," in Proceedings of the $35^{\text {th }}$ International Conference on Machine Learning, JMLR.org vol. 80. Eds. J. Dy and A. Krause(Stockholmsmässan, Stockholm Sweden: PMLR)), 531-540.

Bodla, N., Hua, G., and Chellappa, R. (2018). "Semi-supervised FusedGAN for conditional image generation," in Proceedings of the European Conference on Computer Vision (ECCV), Springer, Cham 669-683.

Budach, S., and Marsico, A. (2018). pysster: Learning Sequence and Structure Motifs in DNA and RNA Sequences using Convolutional Neural Networks. bioRxiv. 34, 3035-3037 doi: 10.1093/bioinformatics/bty222

Chaudhary, K., Poirion, O. B., Lu, L., and Garmire, L. X. (2017). Deep learningbased multi-omics integration robustly predicts survival in liver cancer. Clin. Cancer Res. 24, 1248-1259. doi: 10.1158/1078-0432.ccr-17-0853

Chen, X., Duan, Y., Houthooft, R., Schulman, J., Sutskever, I., and Abbeel, P. (2016). "Infogan: Interpretable representation learning by information maximizing generative adversarial nets," in Advances in Neural Information Processing Systems, vol. 29 . Eds. D. D. Lee, M. Sugiyama, U. V. Luxburg, I. Guyon and R. Garnett (Curran Associates, Inc. in Red Hook, NY) 2172-2180.

Chen, Y., Li, Y., Narayan, R., Subramanian, A., and Xie, X. (2016). Gene expression inference with deep learning. Bioinformatics 32, 1832-1839. doi: 10.1093/ bioinformatics/btw074

Ching, T., Himmelstein, D. S., Beaulieu-Jones, B. K., Kalinin, A. A., Do, B. T., Way, G. P., et al. (2018). Opportunities and Obstacles for Deep Learning in Biology and Medicine. J. R Soc. Interface 15, 141, 1-47. doi: 10.1098/rsif.2017.0387

Creswell, A., Bharath, A. A., and Sengupta, B. (2017). Conditional autoencoders with adversarial information factorization. CoRR. abs/1711.05175.

Duan, Q., Flynn, C., Niepel, M., Hafner, M., Muhlich, J. L., Fernandez, N. F., et al. (2014). LINCS canvas browser: Interactive web app to query, browse and interrogate LINCS L1000 gene expression signatures. Nucleic Acids Res. 42, W449-W460. doi: 10.1093/nar/gku476

Duvenaud, D. K., Maclaurin, D., Iparraguirre, J., Bombarell, R., Hirzel, T., AspuruGuzik, A., et al. (2015). "Convolutional networks on graphs for learning
Zhavoronkov, who is the co-author of the patent covering this technology.

\section{SUPPLEMENTARY MATERIAL}

The Supplementary Material for this article can be found online at: https://www.frontiersin.org/articles/10.3389/fphar. 2020.00269/full\#supplementary-material.

molecular fingerprints," in Advances in Neural Information Processing Systems, vol. 28. Eds. C. Cortes, N. D. Lawrence, D. D. Lee, M. Sugiyama and R. Garnett (Curran Associates, Inc. in Red Hook, NY) 2224-2232.

Gaulton, A., Hersey, A., Nowotka, M., Bento, A. P., Chambers, J., Mendez, D., et al. (2016). The ChEMBL database in 2017. Nucleic Acids Res. 45, D945-D954. doi: $10.1093 /$ nar/gkw1074

Gawehn, E., Hiss, J. A., and Schneider, G. (2016). Deep learning in drug discovery. Mol. Inf. 35, 3-14. doi: 10.1002/minf.201501008

Gómez-Bombarelli, R., Wei, J. N., Duvenaud, D., Hernández-Lobato, J. M., Sánchez-Lengeling, B., Sheberla, D., et al. (2018). Automatic chemical design using a data-driven continuous representation of molecules. ACS Cent. Sci. 4, 268-276. doi: 10.1021/acscentsci.7b00572

Goodfellow, I., Pouget-Abadie, J., Mirza, M., Xu, B., Warde-Farley, D., Ozair, S., et al. (2014). "Generative adversarial nets," in Advances in Neural Information Processing Systems, Curran Associates, Inc. vol. 27. , 2672-2680.

Guimaraes, G. L., Sanchez-Lengeling, B., Farias, P. L. C., and Aspuru-Guzik, A. (2017). Objective-reinforced generative adversarial networks (ORGAN) for sequence generation models. CoRR. abs/1705.10843.

Hu, Q., SzabÃ, A., Portenier, T., Favaro, P., and Zwicker, M. (2018). "Disentangling factors of variation by mixing them," in The IEEE Conference on Computer Vision and Pattern Recognition, vol. 2797. (CVPR).

Kadurin, A., Aliper, A., Kazennov, A., Mamoshina, P., Vanhaelen, Q., Khrabrov, K., et al. (2016). The cornucopia of meaningful leads: Applying deep adversarial autoencoders for new molecule development in oncology. Oncotarget 8, 10883-10890. doi: 10.18632/oncotarget.14073

Kadurin, A., Aliper, A., Kazennov, A., Mamoshina, P., Vanhaelen, Q., Khrabrov, K., et al. (2017a). The Cornucopia of Meaningful Leads: Applying Deep Adversarial Autoencoders for New Molecule Development in Oncology. Oncotarget 8, 10883-10890. doi: 10.18632/oncotarget.14073

Kadurin, A., Nikolenko, S., Khrabrov, K., Aliper, A., and Zhavoronkov, A. (2017b). druGAN: An advanced generative adversarial autoencoder model for de novo generation of new molecules with desired molecular properties in silico. Mol. Pharm. 14, 3098-3104. doi: 10.1021/acs.molpharmaceut.7b00346

Kingma, D. P., and Ba, J. (2015). Adam: A Method for Stochastic Optimization. Int. Conf. Learn. Representations.

Kingma, D. P., and Welling, M. (2013). Auto-encoding variational bayes. CoRR. abs/1312.6114

Kocaoglu, M., Snyder, C., Dimakis, A. G., and Vishwanath, S. (2018). CausalGAN: Learning causal implicit generative models with adversarial training. Int. Conf. Learn. Representations.

Kuzminykh, D., Polykovskiy, D., Kadurin, A., Zhebrak, A., Baskov, I., Nikolenko, S., et al. (2018). 3D Molecular Representations Based on the Wave Transform for Convolutional Neural Networks. Mol. Pharm. 15, 4378-4385. doi: 10.1021/ acs.molpharmaceut.7b01134

Lample, G., Zeghidour, N., Usunier, N., Bordes, A., Denoyer, L., and Ranzato, M. A. (2017). "Fader networks: Manipulating images by sliding attributes," in Advances in Neural Information Processing Systems 30. Eds. I. Guyon, U. V. Luxburg, S. Bengio, H. Wallach, R. Fergus, S. Vishwanathan and R. Garnett (Curran Associates, Inc.), 5967-5976.

Li, Y., Pan, Q., Wang, S., Peng, H., Yang, T., and Cambria, E. (2019). Disentangled variational auto-encoder for semi-supervised learning. Inf. Sci. 482, 73-85. doi: 10.1016/j.ins.2018.12.057

Liu, X. L. (2017). Deep recurrent neural network for protein function prediction from sequence. bioRxiv. doi: 10.1101/103994 
Lusci, A., Pollastri, G., and Baldi, P. (2013). Deep Architectures and Deep Learning in Chemoinformatics: the Prediction of Aqueous Solubility for Drug-like Molecules. J. Chem. Inf. Model 53, 1563-1575. doi: 10.1021/ci400187y

Ma, J., Sheridan, R. P., Liaw, A., Dahl, G. E., and Svetnik, V. (2015). Deep neural nets as a method for quantitative structure-activity relationships. J. Chem. Inf. Model. 55, 263-274. doi: 10.1021/ci500747n. PMID: 25635324.

Makhzani, A., Shlens, J., Jaitly, N., and Goodfellow, I. J. (2015). Adversarial Autoencoders. CoRR. abs/1511.05644. 73, 1482-1490.

Mamoshina, P., Vieira, A., Putin, E., and Zhavoronkov, A. (2016). Applications of deep learning in biomedicine. Mol. Pharma. 13, 1445-1454. doi: 10.1021/ acs.molpharmaceut.5b00982

Mamoshina, P., Kochetov, K., Putin, E., Cortese, F., Aliper, A., Lee, W.-S., et al. (2018a). Population specific biomarkers of human aging: A big data study using south korean, canadian, and eastern european patient populations. Journals Gerontology: Ser. A. 73, 1482-1490. doi: 10.1093/gerona/gly005

Mamoshina, P., Volosnikova, M., Ozerov, I. V., Putin, E., Skibina, E., Cortese, F., et al. (2018b). Machine learning on human muscle transcriptomic data for biomarker discovery and tissue-specific drug target identification. Front. Genet. 9, 242. doi: 10.3389/fgene.2018.00242

Mamoshina, P., Kochetov, K., Cortese, F., Kovalchuk, A., Aliper, A., Putin, E., et al. (2019). Blood biochemistry analysis to detect smoking status and quantify accelerated aging in smokers. Sci. Rep. 9, 142. doi: 10.1038/s41598-018-35704-w

Mathieu, M. F., Zhao, J. J., Zhao, J., Ramesh, A., Sprechmann, P., and LeCun, Y. (2016). "Disentangling factors of variation in deep representation using adversarial training," in Advances in Neural Information Processing Systems, vol. 29. Eds. D. D. Lee, M. Sugiyama, U. V. Luxburg, I. Guyon and R. Garnett (Curran Associates, Inc. in Red Hook, NY) 5040-5048.

Mirza, M., and Osindero, S. (2014). Conditional generative adversarial nets. CoRR. abs/1411.1784.

Ngiam, J., Khosla, A., Kim, M., Nam, J., Lee, H., and Ng, A. Y. (2011). "Multimodal deep learning," in Proceedings of the 28th International Conference on International Conference on Machine Learning, vol. 11. (Omnipress, ICML: 2600 Anderson St, Madison, WI, United States), 689-696.

Ozerov, I. V., Lezhnina, K. V., Izumchenko, E., Artemov, A. V., Medintsev, S., Vanhaelen, Q., et al. (2016). In Silico Pathway Activation Network Decomposition Analysis (iPANDA) as a Method for Biomarker Development. Nat. Commun. 7, 13427. doi: 10.1038/ncomms13427

Perarnau, G., van de Weijer, J., Raducanu, B., and Álvarez, J. M. (2016). Invertible conditional gans for image editing. Neural Inf. Process. Syst. Workshop Adversarial Training.

Polykovskiy, D., Zhebrak, A., Sanchez-Lengeling, B., Golovanov, S., Tatanov, O., Belyaev, S., et al. (2018a). Molecular Sets (MOSES): A Benchmarking Platform for Molecular Generation Models. arXiv preprint arXiv:1811.12823.

Polykovskiy, D., Zhebrak, A., Vetrov, D., Ivanenkov, Y., Aladinskiy, V., Bozdaganyan, M., et al. (2018b). Entangled conditional adversarial autoencoder for de-novo drug discovery. Mol. Pharm. 15, 4398-4405. doi: 10.1021/acs.molpharmaceut.8b00839

Putin, E., Mamoshina, P., Aliper, A., Korzinkin, M., Moskalev, A., Kolosov, A., et al. (2016). Deep Biomarkers of Human Aging: Application of Deep Neural Networks to Biomarker Development. Aging (Albany NY) 8, 1021-1033. doi: 10.18632/aging.100968

Putin, E., Asadulaev, A., Ivanenkov, Y., Aladinskiy, V., Sanchez-Lengeling, B., Aspuru-Guzik, A., et al. (2018a). Reinforced Adversarial Neural Computer for de Novo Molecular Design. J. Chem. Inf. Model 58, 1194-1204. doi: 10.1021/ acs.jcim.7b00690

Putin, E., Asadulaev, A., Vanhaelen, Q., Ivanenkov, Y., Aladinskaya, A. V., Aliper, A., et al. (2018b). Adversarial Threshold Neural Computer for Molecular de Novo Design. Mol. Pharm. 15, 4386-4397. doi: 10.1021/acs.molpharmaceut.7b01137

Qin, Q., and Feng, J. (2017). Imputation for transcription factor binding predictions based on deep learning. PloS Comput. Biol. 13, e1005403+. doi: 10.1371/journal.pcbi.1005403

Sanchez, B., and Aspuru-Guzik, A. (2018). Inverse molecular design using machine learning: Generative models for matter engineering. Science 361, 360-365. doi: $10.1126 /$ science.aat2663

Segler, M. H. S., Kogej, T., Tyrchan, C., and Waller, M. P. (2017). Generating focused molecule libraries for drug discovery with recurrent neural networks. ACS Cent. Sci. 4, 120-131. doi: 10.1021/acscentsci.7b00512
Sohn, K., Lee, H., and Yan, X. (2015a). "Learning structured output representation using deep conditional generative models," in Advances in Neural Information Processing Systems, vol. 28 . Eds. C. Cortes, N. D. Lawrence, D. D. Lee, M. Sugiyama and R. Garnett (Curran Associates, Inc. in Red Hook, NY) 34833491.

Sohn, K., Lee, H., and Yan, X. (2015b). "Learning structured output representation using deep conditional generative models," in Advances in Neural Information Processing Systems, vol. 28 . Eds. C. Cortes, N. D. Lawrence, D. D. Lee, M. Sugiyama and R. Garnett (Curran Associates, Inc.), 3483-3491.

Suzuki, M., Nakayama, K., and Matsuo, Y. (2017). "Joint Multimodal Learning with Deep Generative Models," in International Conference on Learning Representations Workshop.

Wang, W., Arora, R., Livescu, K., and Bilmes, J. (2015). "On deep multi-view representation learning," in Proceedings of the 32Nd International Conference on International Conference on Machine Learning - Volume 37. (JMLR.org), ICML'15. 1083-1092.

Wang, W., Arora, R., Livescu, K., and Bilmes, J. A. (2016a). On deep multi-view representation learning: Objectives and optimization. CoRR. abs/1602.01024.

Wang, W., Lee, H., and Livescu, K. (2016b). Deep variational canonical correlation analysis. CoRR. abs/1610.03454.

Weininger, D., Weininger, A., and Weininger, J. L. (1989). SMILES. 2. algorithm for generation of unique SMILES notation. J. Chem. Inf. Comput. Sci. 29, 97101. doi: 10.1021/ci00062a008

Weininger, D. (1988). SMILES, a chemical language and information system. 1. introduction to methodology and encoding rules. J. Chem. Inf. Comput. Sci. 28, 31-36. doi: 10.1021/ci00057a005

West, M. D., Labat, I., Sternberg, H., Larocca, D., Nasonkin, I., Chapman, K. B., et al. (2018). Use of Deep Neural Network Ensembles to Identify Embryonicfetal Transition Markers: Repression of COX7A1 in Embryonic and Cancer Cells. Oncotarget 9, 7796-7811. doi: 10.18632/oncotarget.23748

Yang, J., Reed, S., Yang, M.-H., and Lee, H. (2015). "Weakly-supervised disentangling with recurrent transformations for 3D view synthesis," in Proceedings of the 28th International Conference on Neural Information Processing Systems, vol. 1. (Cambridge, MA, USA: MIT Press), 1099-1107. NIPS'15.

Zhang, Z., Song, Y., and Qi, H. (2017). Age progression/regression by conditional adversarial autoencoder. IEEE Conf. Comput. Vision Pattern Recogn. (CVPR). 43524360. doi: 10.1109/CVPR.2017.463

Zhao, S., Song, J., and Ermon, S. (2017). InfoVAE: Information maximizing variational autoencoders. CoRR. abs/1706.02262. doi: 10.1609/ aaai.v33i01.33015885

Zhavoronkov, A., Ivanenkov, Y. A., Aliper, A., Veselov, M. S., Aladinskiy, V. A., Aladinskaya, A. V., et al. (2019). Deep learning enables rapid identification of potent ddr1 kinase inhibitors. Nat. Biotechnol., 1-4. doi: 10.1038/s41587-0190224-x

Zhavoronkov, A. (2018). Artificial intelligence for drug discovery, biomarker development, and generation of novel chemistry. Mol. Pharm. 15, 4311-4313. doi: 10.1021/acs.molpharmaceut.8b00930

Zheng, J., Zhang, X., Zhao, X., Tong, X., Hong, X., Xie, J., et al. (2017). DeepRBPPred: Predicting RNA binding proteins in the proteome scale based on deep learning. bioRxiv. 8, 15264 doi: 10.1101/210153

Zhu, J.-Y., Park, T., Isola, P., and Efros, A. A. (2017). Unpaired image-to-image । translation using cycle-consistent adversarial networks. 2017 IEEE Int. Conf. Comput. Vision (ICCV), 2242-2251. doi: 10.1109/ICCV.2017.244

Conflict of Interest: RS, MK, AZ, AK, AA, and DP work for Insilico Medicine, a commercial artificial intelligence company. SN works for Neuromation OU, a company engaged in AI development through synthetic data and generative models.

Copyright $\odot 2020$ Shayakhmetov, Kuznetsov, Zhebrak, Kadurin, Nikolenko, Aliper and Polykovskiy. This is an open-access article distributed under the terms of the Creative Commons Attribution License (CC BY). The use, distribution or reproduction in other forums is permitted, provided the original author(s) and the copyright owner(s) are credited and that the original publication in this journal is cited, in accordance with accepted academic practice. No use, distribution or reproduction is permitted which does not comply with these terms. 